\title{
FUNDAMENTAR PARA CAUTIVAR \\ A propósito de la formación \\ de docentes en tecnología
}

\author{
Edgar Mendoza Parada \\ Profesor Departamento de Tecnología \\ Universidad Pedagógica Nacional \\ edgarmen @colomsat.net.co
}

\begin{abstract}
In order of considerations about different characters of technology and a good justification of its teaching skills lo medium and basic education in terms of its intrinsic intermediate potential, the article advocate good skill of teachers for the area, in a view that collect technology, science and mathematics. The article insists en the necessity of a strong disciplinary formation on those areas to increase the potential of the teacher en technology.
\end{abstract}

\begin{abstract}
RESUMEN
A partir de consideraciones sobre el carácter interdisciplinario de la tecnología y de una justificación de su enseñanza en la educación media y básica en términos de su potencial pedagógico intrínseco, se aboga por la formación de docentes para el área en una perspectiva que integre tecnología, ciencias básicas y matemáticas. Se insiste en la necesidad de una sólida formación disciplinar en éstas áreas para incrementar el potencial pedagógico del docente de tecnología.
\end{abstract}

1. No parece ser necesaria una justificación sobre la necesidad de contenidos científicos en la enseñanza básica. Hace mucho tiempo vienen formándose licenciados en ciencias naturales hacia el ejercicio laboral en grados bien precisos de la escolaridad básica o media. La tradición que consolida temáticas y el prestigio propio de la ciencia parece hacer incuestionable los programas de formación de educadores en sus distintas áreas. La educación en tecnología en la educación media, en cambio, aunque renovada en terminología e influida por la utilización arrolladora de los computadores y por el impacto del mercado informático, ha sido tradicionalmente vista con cierto desprecio desde su origen vocacional que tenía el estigma de la discriminación social. Nació como respuesta laboral para las capas más pobres de la población escolar ante la insuficiente cobertura de la educación superior frente a la expansión de la matricula, y no por sus potenciales bondades educativas. Sus contenidos temáticos nunca tuvieron peso en la admisión universitaria, ni siquiera para ingenierías o carreras científicas.

Con el reconocimiento de la importancia de la educación científica y tecnológica desde la óptica de estrategia política y desarrollo económico autosustentable de unas décadas 
atrás, y con un poco de pragmatismo voluntarista, se llegó incluso a identificar algunos objetivos o resultados deseables de la educación tecnológica de niveles altos con los de la educación técnica del bachillerato. Se proponía, por ejemplo, como objetivos en la educación media técnica, apropiar tecnología o contribuir con soluciones tecnológicas a la demanda de problemas puntuales de comunidades locales, y como deseable, la articulación del currículum con tales demandas, sin ninguna consideración con las potencialidades y limitaciones de los distintos niveles de formación. Así, lo que sin duda puede ser una buena opción como estrategia para la educación cívica, a través de una exigencia de poner conocimientos al servicio de los problemas de la comunidad, se convertía en imperativo educativo a partir del reconocimiento trivial de que la tecnología "solucionar problemas".

Una corriente renovadora no suficiente asimilada, ni formulada de manera que pueda ser medianamente operativa para fines educativos prácticos, tiende a dar más importancia a la componente tecnológica en la educación básica, una vez desprovista de su carácter vocacional. Se sustenta en el hecho de que la tecnología es parte esencial de la cultura contemporánea, y que por tanto debe ser un campo educativo para todos los ciudadanos, independientemente de su futura orientación profesional, y promueve la alfabetización científica tecnológica dentro y fuera del sistema escolar. La educación tecnológica está ahora en los primeros escenarios de la agenda educativa liberada de su lastre instrumental de corto plazo, con un poco más de reconocimiento académico y un notable incremento de investigación sobre su propia didáctica. En años recientes, a muy pocos educadores se les hubiera ocurrido que una instrucción considerada como esencialmente operativa pudiese desarrollar habilidades de pensamiento y que fuera deseable introducirla como una de las componentes en la educación básica para amplios sectores de la población. Mucho menos, para aquellos tradicionalmente acreedores a los mejores destinos académicos.

2. La tecnología vista desde una perspectiva cultural conduce al planteamiento de la alfabetización y divulgación tecnológica, lo que se traduce desde la educación elemental en un esfuerzo para proveer al ciudadano común de un mínimo manejo operativo de su entorno tecnológico, y para facilitarle una comprensión humanista de la tecnología que potencialice su interacción social, por ejemplo, la participación política inteligente en una toma de decisiones en que la variable estrictamente tecnológica pueda ser relevante. Por el impacto cultural de la informática es casi natural que el contenido operativo de los currículos tienda a volcarse sobre el manejo de computadores, en el mejor de los casos en aplicaciones de software educativo o a aplicaciones de uso extendido por el mercado, reduciendo la enseñanza de la tecnología a la "informática". La opción de una comprensión sobre los principios de funcionamiento del instrumental tecnológico más cercano al entorno doméstico parece tanto más difícil en la medida que éste se vuelve más complejo. Sobre lo que pueda considerarse relación ciencia tecnología y sociedad, el espectro de posibilidades temáticas es de suyo considerablemente amplio y de una riqueza que exige el concurso de prácticamente todas las áreas de las ciencias humanas. Son tópicos esenciales a cualquier currículo que pretenda formar docentes en el área y una posibilidad de indiscutible pertinencia social como extensión universitaria.

Sin embargo, al sustentar la formación en tecnología sobre estos argumentos sigue conservándose prioritamente su carácter instrumental si bien que en una perspectiva bastante más global que la del enfoque vocacional. En lo que sigue se trata de explorar una justificación de La enseñanza de la tecnología en términos de su potencial pedagógico intrínseco y se aboga por la formación de docentes para el área en una perspectiva que integre tecnología, ciencias básicas y matemáticas. 
3. Desde el punto de vista curricular, la posibilidad integradora de la tecnología se da tanto por el carácter interdisciplinario propio de cualquier problema de tipo tecnológico, sea de naturaleza explicativa o de construcción, como por el hecho de proporcionar problemas significativos que conducen, en las distintas etapas de desarrollo del estudiante, a abordajes integrados, y a explicaciones coherentes y cerradas con posibilidad de ser tratadas en diferentes niveles de abstracción. Por coherentes y cerradas se quiere significar, potenciadoras de una lógica interna sustentada en redes conceptuales previamente asimiladas en las que inferencias, analogías o interrelaciones adquieren significado, permitiendo en cada estadio de desarrollo del estudiante, comprensiones esencialmente válidas y sólidas para la construcción continua de conocimientos cada vez más complejos y abstractos. Esto es, relativamente alejadas de la simple divulgación científica, caracterizada por explicaciones basadas en descripciones que se apoyan en analogías, y en las que queda mucho por explicar hasta tanto no se disponga del arsenal de abstracción propio de cada disciplina. Por ejemplo, el análisis de los mecanismos asociados a un móvil de niños puede explorarse desde los niveles más básicos de inferencias en relaciones causa efecto, hasta la modelación, resolución matemática y simulación de las ecuaciones del movimiento, en los primeros niveles de la educación universitaria.

Un juego mecánico puede ser más fértil que la descripción de la estructura atómica. Ésta, tratada por ejemplo, a través de modelos planetarios de cuyas limitaciones no necesariamente el docente advierte a sus estudiantes, puede además dejar imágenes completamente falseadas de los modelos aportados por la ciencia, sobre todo si el docente no dispone de un bagaje teórico con niveles de abstracción superiores al que puede ser suficiente para fines descriptivos. En este sentido, en la selección de contenidos primarían aquellos que pueden excitar mas larga y ampliamente la lógica del estudiante, y no los que intrínsecamente puedan ser más importantes.

El problema tecnológico, definido a partir de un objeto o situación deseada, integra saberes disciplinarios específicos en función de una explicación tecnológica usando también teoría tecnológica, normalmente de un nivel operacional mayor que el de las ciencias básicas, como, por ejemplo, modelos de circuitos en vez de problemas de campos electromagnéticos, métodos estandarizados de fuerte tendencia algorítmica, know how, etc. La competencia en tecnología requiere de una disposición interdisciplinar para el análisis de problemas, que va más allá de las habilidades analíticas o creativas y de la competencia disciplinar propiamente dicha. Si se entiende la tecnología en su conjunción de saber, hacer y poder, en el sentido más esencial de capacidad para transformar el mundo, el contexto cultural en que se aplica y desarrolla no es una adición humanista al currículo sino que es inherente a la comprensión del hecho tecnológico. Tecnología es un eje transversal que toca diversas áreas curriculares y es una instancia articuladora no solo de las áreas en las que debe apoyarse para darse sus propias explicaciones internas sino en el ámbito más ampliamente cultural.

4. En esta perspectiva integradora, parece ser necesaria una fuerte reestructuración de los programas que forman docentes para el área de tecnología. El ejercicio de la educación tecnológica en los niveles básicos, si bien que enriquecida con didácticas que se alejan de las prácticas instrumentales y operativas con las que surgió el área, continúan generalmente limitadas a los contenidos tradicionales de electricidad, electrónica, mecánica y diseño, con la excepción de nuevos contenidos en informática, pero en todo caso sin un esfuerzo sistemático de re-programación de los estudios de pregrado. La formación disciplinar parece requerir mayor profundidad, amplitud y versatilidad. La esperanza de que los docentes acometan su tarea de manera 
interdisciplinaria se enfrenta con limitaciones que van desde prácticas administrativas hasta las tradiciones de "especialización" no por la vía de la profundización sino de la carencia de conocimientos en áreas afines, y parece ser una quimera. Sin duda se obtendrán mejores resultados si la aspiración a un trabajo interdisciplinario se formula explícitamente en la programación de contenidos y se implementaran estrategias pedagógicas acordes. El esquema de departamentos aislados en que tradicionalmente se organizó la educación superior, tiene en instituciones pequeñas la doble desventaja del aislamiento académico y el desaprovechamiento de los limitados recursos humanos.

Un docente de tecnología con capacidad para transitar transversalmente por el currículo de la educación básica y media, precisaría de una formación interdisciplinar mucho más rica que la proporcionada en la actualidad. Aunque existe un discurso reiterativo de todos los matices sobre la importancia de la educación tecnológica, ampliamente difundido, los rumbos temáticos para niveles básicos son equívocos e imprecisos como corresponde a un área que se encuentra en fase de consolidación en todo el mundo. Las directrices del MEN no llegan a un nivel explotable en términos de permitir introducir cambios procedimentales que impacten masivamente el aula de clase. Las dificultades para implementar reformas tienen que ver no solo con la insuficiente definición de un proceso que apenas se vislumbra sino con la ausencia correlativa de programas de formación profesional o en ejercicio, coherentes con las propuestas planteadas. El obstáculo crítico con el que se enfrentan las reformas mejor intencionadas parece estar en la insuficiente rapidez y calidad de la respuesta por parte de los ejecutores, lo que tiende con el tiempo a desvirtuar los propósitos originales, y en la limitada capacidad para presentar de manera autónoma visiones alternativas.

Perdida la respuesta vocacional, la visualización de un horizonte para el área se ha dirigido a la búsqueda de una identidad respondiendo a la pregunta fundamental de lo que se entiende por tecnología y cuál sería el objetivo de una enseñanza no profesionalizante, vía una diferenciación con la ciencia. El camino no parece haber sido muy fértil, y a partir de definiciones estándar de ciencia y tecnología se han producido no pocos equívocos, como el de buscar un método universal para la tecnología análogo al mítico "método científico" y darle ese estatus al diseño. Un esfuerzo de afianzamiento de la formación disciplinar al tiempo con una indagación por las potencialidades pedagógicas propias de la enseñanza tecnológica y una búsqueda de claridad filosófica sobre el área podría ser un camino más fértil. Las componentes tecnológicas de la educación básica y la definición de un currículo para la formación de docentes deben estar mutuamente imbricados. Puede ser táctico para propósitos de diseño curricular restarle importancia temporalmente a la búsqueda de la diferenciación entre ciencia y tecnología.

No solamente el concepto de tecnociencia parece muy atractivo sino que la separación de habilidades en teóricas y prácticas perdió vigencia con la misma superación del enfoque vocacional. "En todas partes y en todos los tiempos, ha habido hombres prácticos, absortos en "irreductibles y obstinados hechos". Por todo el mundo en todas partes y en todos los tiempos ha habido hombres de temperamento filosófico absortos en el entretejer de los principios generales. Esta unión de un apasionado interés por los pequeños detalles con una idéntica pasión por la generalización abstracta es lo que constituye la novedad de nuestra sociedad actual". En la formación básica y aún en la formación universitaria esos talentos se dan mezclados. Tanto más rica una formación si logra maravillar con los fundamentos y consecuencias de la mecánica cuántica como emocionar con una simulación de un sistema de control. Escoger tempranamente un enfoque priva al estudiante de desarrollar sus potencialidades en una u otra dirección, y de elegir la opción profesional y vital con argumentos más maduros. 
El poco tránsito entre las ciencias y las ingenierías en nuestra educación superior puede explicarse por una excesiva "feudalización" de los programas y experiencias académicas presentadas a los estudiantes y en una serie de prejuicios que asocian profundidad de conocimiento con nomenclaturas profesionales.

Pero para la conformación de un currículo en tecnología en una óptica ínter-disciplinar, además de las limitaciones administrativas impuestas por la tradición y las derivadas de una formación en demasía específica y no pocas veces limitada tanto en amplitud de contenidos como en nivel de profundidad, el trabajo interdisciplinario se enfrenta con problemas de orden epistemológico. En presencia de un conflicto metodológico, no es del todo claro qué enfoque disciplinario privilegiar ni para la construcción del currículo ni para un proyecto temático específico. En ese sentido el conflicto puede solucionarse mejor en la esfera más pragmática de la conciliciación.

Las áreas profesionales que se han establecido con una amplia tradición permiten definir criterios para enfoques y pertinencias temáticas en un currículo, aunque no exista un referente de reflexión epistemológica o de fundamentación de la disciplina fuerte y explícito. No es este el caso de la formación de docentes en tecnología, cuando la misma actividad deseable del docente está en proceso de discusión, definición, elaboración y asentamiento en el mundo entero. Este es uno de los puntos para una discusión más abierta en la conformación de un currículo. Los caminos no están prescritos en parte alguna. Pero algunos errores de tipo operativo que se han de evitar si es fácil vislumbrarlos: evidentemente no se trata de establecer una confederación de feudos disciplinares, y el primer riesgo es el de querer introducir en un nuevo programa académico; por sucesivas adiciones, todo el conocimiento que a juicio de los especialistas de cada disciplina es imprescindible para que su propio contenido disciplinar no quede incompleto o insustancial. Una aproximación interdisciplinaria cobra sentido y evidencia toda su fuerza en relación con un proyecto. La manera de integrar los conocimientos se define a partir del proyecto y para el caso de la formación de docentes ese proyecto es el de la educación tecnológica básica y media. La conformación de currículos para la formación básica en tecnología y para la formación de docentes deben ser paralelos y complementarios. El de la formación de docentes por tratar-se de niveles de abstracción mayores puede recurrir a un abordaje más disciplinar. El de la formación básica privilegia características psicológicas del niño. Un punto de enlace, fuente de ajustes y de investigación permanente se podría derivar de la experiencia proporcionada por las prácticas docentes.

5. En la explicación de un objeto o una situación tecnológica se manifiesta el carácter interdisciplinario o multidisciplinario de la tecnología. Las analogías entre distintos sistemas técnicos, hidraúlicos, térmicos, eléctricos pueden ser claramente definidas basándose en el hecho de que las ecuaciones diferenciales que describen su dinámica son del mismo tipo y por lo tanto lo analizado para una variable pueden re-interpretarse en diferentes contextos mostrándose el comportamiento análogo. La analogía no es simplemente formal como pueden serlo las encontradas entre sistemas físicos y económicos, sino que obedecen a los mismos principios físicos fundamentales mirados desde niveles de abstracción superiores, y se pueden derivar lógicamente de tales principios. La visión unificadora que proporciona un enfoque sistémico, usada por ejemplo en teoría de control, tiene un potencial didáctico enorme pues pone de manifiesto nociones comunes a muchos sistemas técnicos, como flujos de energía, fenómenos de transporte, flujos de información, realimentación, estabilidad, etc. La matemática se torna fundamental. 
Matemáticas, ciencia y tecnología están fuertemente interrelacionadas; sus fronteras son artificiales y desactualizadas. Tópicos frontera frecuentemente reconocen como necesaria la interdisciplinariedad y complementariedad de las disciplinas a la hora de entender y resolver problemas científico-tecnológicos cada vez más complejos y entremezclados. Sin embargo, la disponibilidad a la aceptación de la importancia del conocimiento matemático no es la norma en los programas de las licenciaturas tecnológicas y no pocas veces el ambiente a la introducción de sus tópicos, es abiertamente hostil.

La crítica a la acción de dictar clase, como patrón modelo básico de la enseñanza se ha convertido en un lugar común de muy buen recibo, y en el más socorrido preámbulo a cualquier "propuesta alternativa" de tipo didáctico. Un mayor impacto y aceptación general es obtenida si se personifica en el profesor de matemáticas tipificado escribiendo permanentemente casi de manera compulsiva sobre un tablero, desarrollos teóricos o ejercicios. Aunado a las críticas sobre falta de participación del estudiante, "carencia de utilidad", o a la "excesiva teorización" aún en los programas de formación profesional donde cierto conocimiento matemático es considerado fundamental, y al carácter intimidatorio de las evaluaciones en las que en el fondo pareciera jugarse el prestigio personal, la formación matemática parece ser sobrellevada en las carreras técnicas y algunas científicas casi como un trago amargo que afortunadamente solo es necesario sufrir en los primeros semestres.

Una crítica más fundamentada desde la enseñanza de la física señala que tópicos desarrollados sobre una base matemática exclusivamente formal no garantizan una comprensión de las teorías o de los conceptos, siendo típico el ejemplo de los cursos de electromagnetismo, en los que se puede someter al estudiante a engorrosos problemas de cálculo vectorial para calcular campos eléctricos 0 magnéticos en diferentes disposiciones geométricas, o a derivaciones formales a partir de las leyes de Maxwell, sin que el estudiante con esto vislumbre las características fundamentales de la teoría. Esto puede ser parafraseado en términos de competencias, diciendo que la competencia lingüística 0 , más ampliamente, comunicativa no implica la competencia disciplinar. Limitaciones del enfoque formalista y operativo se hacen evidentes en las tradicionales evaluaciones en el que se exige la solución de ejercicios siempre asociados a un capitulo particular; nunca un ensayo o una solución de problemas prácticos o teóricos no necesariamente referenciados a lo estudiado previamente. Una expresión de la tradición formalista parece ser la iniciación de la enseñanza de la física solo hasta los últimos grados de la enseñanza media cuando el estudiante dispone de una base operatoria para la utilización de una cierta cantidad de "fórmulas" y la importancia fundamental dada a esa capacidad para tratar los tópicos. Sin embargo, en la formación de los docentes, estas limitaciones no se superan suprimiendo la capacitación operatoria matemática sino introduciéndola en un enfoque mas amplio y propiciando situaciones problemáticas teóricas o prácticas en las que las integralidad del conocimiento sea necesaria y la fuerza explicativa de la teoría se manifieste poderosa. En términos de competencias, generando situaciones en las que las competencias pueden emerger. Pero, por otra parte, las limitaciones de competencias comunicativas inhiben el desarrollo de las competencias disciplinares. Un caso patético ilustrativo es el del estudiante de últimos semestres que, accediendo a bibliografía sobre un tema de su particular interés profesional, no puede leerla porque "tiene mucha matemática". Estamos en presencia de un analfabeto funcional frente a los conocimientos en los que se le certifica su especialidad disciplinar, y al que se le ha truncado su potencial para caminar por cuenta propia. 
Por supuesto, actualmente la competencia comunicativa para abordar problemas en los programas de tecnología excede en mucho la habilidad formal matemática y no se supera solo en ese ámbito. La noción de ecosistema comunicativo puede ser muy fértil al respecto. Es necesaria la alfabetización visual que potencie la comunicación a partir de toda clase de representaciones gráficas e imágenes no solo referenciales sino asociadas a procesos. También en este contexto de ampliación del ecosistema comunicativo, deben ser considerados los programas de computador de modelaje de diverso tipo en los que el esfuerzo de programación es mínimo frente al potencial explicativo suministrado.

El uso de paquetes computacionales con rutinas para solucionar problemas matemáticos específicos tiene una justificación intrínseca en la potenciación de la capacidad de cálculo, y en la facilidad para obtener representaciones gráficas de gran valor ilustrativo. Pero además de esto, la posibilidad de solucionar problemas de una manera rápida invocando rutinas, prácticamente sin ningún trabajo de programación y sin conocimiento sobre los métodos numéricos de solución, ofrece la posibilidad de explorar y ejemplificar con "experimentación numérica de esfuerzo mínimo", la riqueza de los conceptos y de las teorías. Desde un punto de vista educativo, esto significa tener a disposición una enorme fuente donde crear motivos de análisis, pero que exige para su exploración, un creciente conocimiento matemático. Para un estudiante de física o de tecnología tiene que ser retador y estimulante, constatar que es más de matemáticas o de física y menos de "computación" lo que se requiere para explotar esas potencialidades. Y para cualquiera que esté en lides educativas sería una pena no usar recursos tan útiles que ya son simples y cotidianos productos de difusión comercial masiva.

La formación de docentes en tecnología con una perspectiva más teórica que la actual, que posibilite una proyección hacia niveles más altos de abstracción sobre la base de una fundamentación matemática más sólida rendiría seguros frutos pedagógicos. Permitiría a los docentes construir conocimientos científico-tecnológicos en forma comprensiva y así poder realizar transposiciones didácticas que favorezcan la comprensión a sus alumnos. Por ejemplo, una pregunta natural que surge de una observación espontánea del entorno tecnológico, indaga sobre la manera como se produce la electricidad. Una respuesta típica de naturaleza descriptiva muestra los distintos tipos de transformación de energía en las centrales eléctricas o recurre a la demostración ilustrativa estándar del laboratorio de física o de los museos de ciencias. Pero aunque es evidente que el docente no podrá sustentar sus explicaciones sobre la base de manipulaciones matemáticas con las leyes de Maxwell, si es razonable que aquel que tenga la capacidad de leer conceptualmente (no simplemente de operar con) lo que las expresiones matemáticas de las leyes describen, dispone de una solvencia comunicativa que lo ibera de la fórmula explicativa ilustrada por el texto de referencia o por la práctica de laboratorio demostrativa estándar. En el desarrollo de sus explicaciones el docente puede estar pensando e interpretando los conceptos de derivada, integral de línea, flujos, campos vectoriales, etc., mientras construye sus propias explicaciones. Su profundidad y claridad conceptual se traducirá necesariamente en mayor habilidad y autonomía explicativa. Podrá mostrar cómo se entrelazan y justifican los conceptos, cómo los fenómenos se manifiestan en el contexto global de una teoría, y se potenciará su capacidad para problematizar el conocimiento y discutir alternativas tecnológicas del mismo.

6. El impacto de la tecnología sobre la sociedad contemporánea no se reduce a las enormes modificaciones que ocasiona la masificación de sus productos, ni al impacto ambiental, sino que se ha tornado ideología y ha invadido toda la cultura. La ciencia y la tecnología se han constituido en una unidad casi autónoma que transfiere a la sociedad sus valores, métodos y finalidades. Si no el mecanismo fundamental, si por lo menos un 
medio a través del cual se efectúa esa transferencia es el aparato educativo, y muy particularmente los educadores de áreas tecnológicas. Una valoración ética no aparece aquí, como algo que se le adicione a la actividad tecnológica o a los planes de estudio a manera de una concesión especial a la "formación integral" a través de unos cuantos cursos de "humanidades" sino que es inherente a la misma gnoseología de la tecnología.

La cultura tecnológica no se consigue solamente facilitando el acceso y familiarizando al estudiante con el más moderno instrumental doméstico, o ilustrándolo sobre sus "principios de funcionamiento" o sobre grandes sistemas técnicos como los de energía, comunicaciones y transporte, sino haciéndolo participe de la racionalidad tecnológica. $Y$ de una ética de responsabilidad social. Ambas, la racionalidad y la ética deberían estar explicitadas en un proyecto educativo institucional de formación científico-tecnológica de los docentes. Esto es necesario, tanto por la expectativa de proseguir estudios en la línea científica o tecnológica como por el hecho de que esa racionalidad ya está instalada en la vida cotidiana.

Finalmente, así como a la actitud positiva frente al conocimiento científico suele atribuírsele en consecuencia, la formación de ciertos valores deseables, a la educación tecnológica puede asociársele otro tanto. Mirado por el lado más positivo la asimilación de valores y habilidades fundamentales a la dinámica laboral de la industria moderna serían altamente deseables; responsabilidad, precisión, trabajo en equipo, organización del trabajo orientada a objetivos, planeación, responsabilidad ante la colectividad en la toma de decisiones, atención a la calidad, son algunos ejemplos.

La ciencia y la tecnología están altamente matematizadas en un doble sentido instrumental operativo y de estructura. Sin embargo desde el punto de vista de la educación en tecnología no es suficiente, por ejemplo, con comprender el concepto de movimiento ni conocer la estructura matemática de las ecuaciones que lo describen; es necesario saber cómo producirlo y controlarlo. La dualidad generalidad, a través de una fuerte capacidad de abstracción, y particularidad, en el sentido de la concreción en el detalle en que se manifiesta una solución técnica en operación, aparece como un compromiso pedagógico y como una fuente de rigor intelectual. En la tecnología aparece, quizás con mayor vigor que en otras disciplinas la necesidad de generalización y discriminación como dos procesos de aprendizaje complementarios.

Generalización, en tanto la economía de pensamiento, temas claves, hechos desencadenantes, y abstracción como recurso esencial para su expresión. La generalización, a través de la fuerte intervención del docente permite que el alumno elabore esquemas, categorizaciones, conceptos inclusores, organizadores previos, ideas fuerza que le ayuden a pensar y comprender, y a aprender a aprender. Particularidad, porque una solución operando es la conjunción de multitud de detalles bien logrados y porque generalizaciones que no se apoyan en la abstracción y en el esfuerzo individual y constante de búsqueda de interrelaciones, frecuentemente son vacías, pedantes y carentes de rigor. En última instancia la formación académica debe inculcar el pudor intelectual. $Y$ con este pudor, y dentro de un ambiente de responsabilidad académica, como manifestación de una más amplia responsabilidad política, debe mantener siempre la aspiración de proporcionar al alumno las herramientas intelectuales o conceptuales, procedimentales y actitudinales que le otorguen la suficiente autonomía personal para seguir construyendo y actualizando su propio conocimiento. 


\section{BIBLIOGRAFÍA}

Acero S. Eduardo; Aparicio F. Juan M. La tecnología una dimensión de la cultura. Madrid: Editepsa, 1988.

Acevedo D., J. A. "La tecnología en las relaciones CTS. Una aproximación al tema" Enseñanza de las ciencias. 1996, 14(1), 35-44.

Acevedo D., José A. "Análisis de algunos criterios para diferenciar entre ciencia y tecnología". Enseñanza de las ciencias. 1998, 16(3), 409-420.

Fleming, R., W. High school graduates' beliefs about science-technologysociety- II The interaction among science, sience, technology and society. Science education.71 (2) 163-186.

Fourez, G. Alfabetización científica y tecnológica. Buenos Aires: Ediciones Colihue. 1994.

Gilbert, J. K. "Educación en tecnología: una nueva asignatura en todo el mundo". Enseñanza de las ciencias, 1 995,13(1), 15-24.

Gilbert,J.K. "The interface between science education and technology education" International Journal of Science Education. 1992. Vol. 14, No. 5, pp.563-578.

Gómez C., Víctor. M. La educación tecnológica en Colombia. Bogotá: EUN.1995.

Gutiérrez. Eduardo. Competencias, competencia comunicativa y alfabetización visual. Seminario Consolidación de proyectos pedagógicos y la formulación de competencias en ciencia y tecnología. Bogotá: ACAC. 2000.

Horkheimer, M. Crítica de la razón instrumental. Buenos Aires: Ediciones Sur. 1969.

Ladriere. ,J. El reto de la racionalidad. La ciencia y la tecnología frente a las culturas. Salamanca: Ediciones Sígueme. 1978.

MEN. Educación en Tecnología: propuesta para la educación básica. Bogotá.1996.

Pessoa de Carvalho, Anna M. GIL Pérez, Daniel. Formaçáo de professores de ciéncias. Sáo Paulo Cortez. 1993.

Padilla, Hugo. "Los objetos tecnológicos. Su base gnoseológica". En: La filosofía y la ciencia en nuestros días. México: Grijalbo. 1976.

Padilla, Hugo. "Las revoluciones conceptuales en la tecnología". La filosofía y las revoluciones científicas. México: Grijalbo. 1977. 